\title{
Effect of Different Herbicides on Growth and Yield of Wheat (Triticum aestivum L.)
}

\author{
A. C. Shivran ${ }^{1}$, Sarita $^{2}$, J. Choudhary ${ }^{1}$ and Jitendra Singh Bamboriya ${ }^{3}$ \\ ${ }^{1}$ Department of Agronomy, Maharana Pratap University of Agriculture \\ and Technology, Udaipur, India \\ ${ }^{2}$ Department of Agronomy, Agriculture University, Jodhpur, India \\ ${ }^{3}$ Department of Soil science, Maharana Pratap University of Agriculture \\ and Technology, Udaipur, India \\ *Corresponding author
}

\section{A B S T R A C T}

Keywords

Herbicides,

Growth,

Wheat, Yield

Article Info

Accepted:

07 March 2020

Available Online:

10 April 2020
A field experiment was conducted to study the Effect of different herbicides on growth and yield of wheat on clay loam soil at Instructional Farm of Rajasthan College of Agriculture, Udaipur during the rabi season of 2016-17. The experiment was laid out in randomize block design has replicated thrice. The experiment consisted of eleven treatments viz. halauxifen methyl ester + florasulam + polyglycol at $12.76 \mathrm{~g} \mathrm{ha}^{-1}\left(\mathrm{~T}_{1}\right)$, metsulfuron + surfactant at $4 \mathrm{~g} \mathrm{ha}^{-1}$ $\left(\mathrm{T}_{2}\right)$, carfentrazone at $20 \mathrm{~g} \mathrm{ha}^{-1}\left(\mathrm{~T}_{3}\right), 2,4-\mathrm{D}$ Na Salt at $500 \mathrm{~g} \mathrm{ha}^{-1}\left(\mathrm{~T}_{4}\right), 2$, 4-D Ester at $500 \mathrm{~g} \mathrm{ha}^{-1}\left(\mathrm{~T}_{5}\right)$, metsulfuron + carfentrazone + surfactant at $4 \mathrm{~g}+20 \mathrm{~g} \mathrm{ha}^{-1}\left(\mathrm{~T}_{6}\right)$, 2, 4-D Na + carfentrazone at $400 \mathrm{~g}+20 \mathrm{~g} \mathrm{ha}^{-1}\left(\mathrm{~T}_{7}\right), 2,4-\mathrm{D} \mathrm{E}+$ carfentrazone at $400 \mathrm{~g}+20 \mathrm{~g} \mathrm{ha}^{1}{ }^{1}\left(\mathrm{~T}_{8}\right)$, halauxifen + florasulam + carfentrazone + surfactant at $10.21 \mathrm{~g}+20 \mathrm{~g} \mathrm{ha}^{1}\left(\mathrm{~T}_{9}\right)$, weedy check $\left(\mathrm{T}_{10}\right)$ and weedy free $\left(\mathrm{T}_{11}\right)$. Among the herbicidal treatments, application of halauxifen + florasulam + polyglycol at 12.76 $\mathrm{g}$ ha- ${ }^{1}$ increased plant height $(41.33,84.27$ and $89.47 \mathrm{~cm})$ at $30,60 \mathrm{DAS}$ and harvest, dry matter accumulation (160.0, 269.4 and $325.0 \mathrm{~g}$ metre ${ }^{-1}$ row length) at $60 \& 90$ DAS and harvest, crop growth rate, grain and straw yield over rest of the weed control treatments.

\section{Introduction}

Wheat is one of the most important crops of India not only in terms of acreage, but also in terms of its versatility for adoption under wide range of agro-climatic conditions and crop growing situations. Wheat is also used for manufacturing of bread, flakes, cakes, biscuits etc. It is produced in wide range of climatic environments and geographic regions (Dixon et al., 2009). It provides 21 percent of the food calories and 20 percent of protein for 
more than 4.5 billion people in 94 developing countries (Braun et al., 2010). Due to rising demographic pressure, it become necessary to augment the productivity of food crops including wheat on continues basis to ensure food security (Swaminathan and Bhawani, 2013). Wheat stands second to rice under food grain with a production of 98.38 million tonnes from an area of 31.8 million ha with a productivity of 3.09 tonnes $\mathrm{ha}^{-1}$ during 201819 (Department of Agriculture, Cooperation \& Farmers Welfare, 2018-19).

The five major wheat growing states of Uttar Pradesh, Punjab, Madhya Pradesh, Haryana and Rajasthan contributed nearly 86.0 per cent of the total production in the country. Punjab has the highest average productivity of $4.70 \mathrm{t} \mathrm{ha}^{-1}$ followed by Haryana (4.40 $\left.\mathrm{t} \mathrm{ha}^{-1}\right)$. Rajasthan accounted for about 10.71 per cent $(3.10 \mathrm{~m} \mathrm{ha})$ of the national area and 11.10 per cent $(10.46 \mathrm{~m} \mathrm{t})$ of grain production with average productivity of $3.1 \mathrm{t} \mathrm{ha}^{-1}$ (Commission for Agricultural Costs and Prices, 2019).Weeds adversely affect the crop growth and yield by competing with crops for limiting resources such as light, water and nutrients (Harper, 1977; Swanton et al., 2015).

The intensity and duration of the crop-weed competition determines the magnitude of crop yield losses (Swanton et al., 2015). Uncontrolled growth of weeds on an average caused about 48 per cent reduction in grain yield of wheat when compared with weed free condition (Singh et al., 2012). Herbicides play an important role for weed control in closely spaced crops like wheat and barley, where manual or mechanical weeding is difficult (Yaduraju and Das, 2002). Among different weed management practices, chemical weed control preferred because of less labor involvement and no mechanical damage to the crop that happens during manual weeding (Marwat et al., 2008). These necessitate evolving a strategy to screen out more herbicides to control the weed flora economically in the wheat fields on large scale. In India, herbicide shares only about 8 per cent of total pesticide consumption in country and we use an average of only about 35 gram herbicides $\mathrm{ha}^{-1}$ annum $^{-1}$ (Gupta, 2007).

\section{Materials and Methods}

The experiment was conducted at the Instructional Farm, Department of Agromomy, Rajasthan College of Agriculture, Udaipur which is situated at $24^{0} 35^{\prime} \mathrm{N}$ latitude and $74^{0} 42^{\prime} \mathrm{E}$ longitude. The region falls under the Agro Climatic Zone IVa of Rajasthan (i.e. Sub-Humid Southern Plain and Aravalli Hills). The average rainfall of Udaipur is $637 \mathrm{~mm}$, most of which (80-85 \%) is received through south-west monsoon during July to early September. The mean maximum and minimum humidity at Udaipur fluctuate in between 46.0 to 92.14 per cent and 10.86 to 47.0 per cent, respectively.

Maximum and minimum temperature during the experimental period ranged between 20.91 ${ }^{\circ} \mathrm{C}$ to $38.14^{\circ} \mathrm{C}$ and $5.64^{\circ} \mathrm{C}$ and $19.74^{\circ} \mathrm{C}$, respectively. There was $3.0 \mathrm{~mm}$ rainfall received during the crop season. The soil of the experimental site was clay loam in texture and slightly alkaline in reaction. It is low in available nitrogen $\left(287.5 \mathrm{~kg} \mathrm{ha}^{-1}\right)$, medium in organic carbon $(0.62 \%)$ and phosphorus $(23.6$ $\mathrm{kg} \mathrm{ha}{ }^{-1}$ ) and high in available potassium (366.1 $\left.\mathrm{kg} \mathrm{ha}^{-1}\right)$.

The experiment was laid out in Randomize Block Design and replicated thrice. Wheat variety Raj.- 4079 was used as test crop. The experiment consisted of eleven treatments viz. halauxifen methyl ester + florasulam + polyglycol at $12.76 \mathrm{~g} \mathrm{ha}^{-1}\left(\mathrm{~T}_{1}\right)$; metsulfuron + surfactant at $4 \mathrm{~g} \mathrm{ha}^{-1}\left(\mathrm{~T}_{2}\right)$; carfentrazone at 20 $\mathrm{g} \mathrm{ha}^{-1}\left(\mathrm{~T}_{3}\right) ; 2$, 4-D Na Saltat $500 \mathrm{~g} \mathrm{ha}^{-1}\left(\mathrm{~T}_{4}\right) ; 2$, 
4-D Esterat $500 \mathrm{~g} \mathrm{ha}^{-1}\left(\mathrm{~T}_{5}\right)$; metsulfuron + carfentrazone + surfactantat $4 \mathrm{~g}+20 \mathrm{~g} \mathrm{ha}^{-1}$ $\left(\mathrm{T}_{6}\right) ; 2,4-\mathrm{D} \mathrm{Na}+$ carfentrazone at $400 \mathrm{~g}+20$ $\mathrm{g} \mathrm{ha}^{-1}\left(\mathrm{~T}_{7}\right) ; 2,4-\mathrm{D} \mathrm{E}+$ carfentrazone at $400 \mathrm{~g}+$ $20 \mathrm{~g} \mathrm{ha}^{-1}\left(\mathrm{~T}_{8}\right)$; halauxifen + florasulam + carfentrazone + surfactant at $10.21 \mathrm{~g}+20 \mathrm{~g}$ ha $^{-1}\left(\mathrm{~T}_{9}\right)$; weedy check $\left(\mathrm{T}_{10}\right)$; weedy free $\left(\mathrm{T}_{11}\right)$. A blanket dose of clodinafop $60 \mathrm{~g}$ ha- 1 was applied 7 days before application of herbicidal treatments to control grassy weeds. Weed free plots were completely weed free from crop germination to harvest by manual weeding.

All the herbicides were applied as postemergence at 32 days after sowing. Ready mix herbicides were applied as per treatment through knapsack sprayer fitted with flat fan nozzle using spray volume of 500 litre $\mathrm{ha}^{-1}$ after calibration. Wheat variety Raj 4079 was drilled at $20.0 \mathrm{~cm}$ row spacing on $11^{\text {th }}$ November, 2016 using $100 \mathrm{~kg} \mathrm{ha}^{-1}$ seed rate. The crop was supplied $120 \mathrm{~kg} \mathrm{~N}^{-1}$ half of which was drilled in crop rows at sowing while remaining half was top dressed in two equal splits at the time of first and second irrigation.

Height of five randomly selected plants in each plot was measured at 30,60,90 DAS and at harvest from the soil surface to fully opened top leaf of the plant before ear emergence and up to the top of upper spikelet (excluding awns) at harvest and mean height was worked out in $\mathrm{cm}$. Dry matter accumulation was recorded at 30, 60, 90 DAS and at harvest.

Two plant samples were harvested from a row length of $25 \mathrm{~cm}$ in the second row on either side in each plot. These samples were first sun dried and then oven dried at $70^{\circ} \mathrm{C}$ till a constant weight was attained. After drying, the samples were weighed for computing the dry weight of plants metre ${ }^{-1}$ row length.

\section{Results and Discussion}

\section{Effect of herbicides on growth parameters}

In general, the aforesaid improvements seems to be on account of their direct impact through least crop-weed competition while, indirect effect might be on account of least competition for plant growth inputs viz. light, space, water and nutrient etc. (Fakkar and Amin, 2012). Thus, under least crop-weed competition, adequate availability of light, optimum temperature, space along with improvement in physiological and morphological characters of the plants can be reasoned for greater photosynthetic rate there by more accumulation of dry matter (Duncon, 1971).

The weed control also resulted in significantly higher LAI which might be due to greater area available for expansion due to weed control for an efficient use of solar radiation by crop, the greater part of the radiation must be absorbed by photosynthetic tissue i.e. leaves. Greater the LAI greater is the ability of plant to produce photosynthates which is converted into greater dry matter (Carlos et al., 2012). All herbicidal weed control treatments significantly increased plant height of crop plants compared to weedy check.

The tallest plants $(41.33,84.27$ and $89.47 \mathrm{~cm})$ at 30,60 DAS and harvest were obtained with the application of halauxifen + florasulam + polyglycol while other treatments are lagged behind. Accumulation Compared to weedy check, these weed control treatments resulted in 12.7 and 12.5 per cent increase in plant height at harvest. Maximum crop dry matter accumulation (160.0, 269.4 and $325.0 \mathrm{~g}$ metre $^{-1}$ row length) at $60 \& 90$ DAS and harvest was recorded with application of halauxifen + florasulam + polyglycol. Various weed control treatments resulted in significant increase in dry matter accumulation ranging 
from 28.9 per cent under the effect of 2, 4-D $\mathrm{Na}$ salt to 44.6 per cent under the effect of halauxifen + florasulam + polyglycol at 60 DAS (Table 1). Maximum number of tillers 0.5 metre row length was achieved by weed free plot (50.0). However, it was at par with halauxifen + florasulam + polyglycol (47.0), halauxifen + florasulam + polyglycol + surfactant (47.0) and 2, 4-D E+ carfentrazone (45.00), representing 42.9, 34.3, 34.3 and 28.6 per cent increase in total tillers metre ${ }^{-1}$ row length at 60 DAS. These seven treatments illustrated 31.2, 27.7, 24.8, 22.4, 22.3, 19.1 and 17.0per cent increase in total tillers 0.5 metrerow length, respectively over weedy check at 90DAS (Table 2). Treatment halauxifen + florasulam + polyglycol, halauxifen + florasulam + carfentrazone + surfactant, 2, 4-D E + carfentrazone, metsulfuron + carfentrazone + surfactant and 2, 4-D Na + carfentrazone accounted for 28.8, $26.9,25.0,23.0,21.1$ and 19.2 per cent increase in total tillers 0.5 metre row length, respectively over weedy check (Table 2 ).

\section{Effect of herbicides on relative growth rate and crop growth rate}

Data revealed that that different herbicides could not affect the RGR significantly; however its ranges from 0.017 to 0.020 between 60-90 DAS (Table 2). However, the maximum relative growth rate $\left(0.036 \mathrm{~g} \mathrm{~g}^{-1}\right.$ day $^{-1}$ ) in weed free plot. Compared to weedy check, all the treatments are statistically at par to each other. Among herbicide treatments, halauxifen + florasulam + polyglycol attain maximum relative growth rate between 30-60 DAS. An examination of data (Table 2) clearly indicated that weed control treatments significantly affect the CGR and recorded the maximum CGR under weed free (177.22 $\mathrm{g} \mathrm{m}^{-}$ ${ }^{2}$ day) followed by halauxifen +florasulam + polyglycol (174.44 $\mathrm{g} \mathrm{m}^{-2}$ day) and all the treatments are at par between 30-60 DAS and maximum CGR observed in weed free $\left(185.78 \mathrm{~g} \mathrm{~m}^{-2}\right.$ day $\left.^{-1}\right)$ which was at par with all the treatments between 60-90 DAS.

\section{Effect of herbicides on visual phytotoxicity score}

A perusal of visual phytotoxicity scoring revealed that at 10 days after spray carfentrazone gave setback to wheat crop by causing Injury more persistent but more persistent injury to wheat plants putting the plants under doubtful recovery zone. Metsulfuron + carfentrazone + surfactant and 2, 4-D Na + carfentrazone resulted in injury more pronounced but not persistent injury at this stage and onwards days. At this stage 2, 4-D E + carfentrazone, and halauxifen + florasulam + carfentrazone caused slight injury and discolouration of wheat plants (Table 3). A perusal of visual scoring revealed that at 10 days after spray carfentrazone gave setback to wheat crop by causing moderate leaf yellowing and this stage recovery possible. Metsulfuron + carfentrazone + surfactant, 2, 4-D E + carfentrazone and 2, 4-D Na + carfentrazone resulted in leaf yellowing more pronounced but not persistent leaf yellowing at this stage and onwards days. At this stage halauxifen + florasulam + carfentrazone caused slight discolouration of wheat plants (Table 3 ).

\section{Effect of herbicides on yield}

The data revealed (Table 4) that minimum gain was recorded by weedy check $(4097 \mathrm{~kg}$ $\mathrm{ha}^{-1}$ ). Amongst treatments, highest yield $\left(5287 \mathrm{~kg} \mathrm{ha}^{-1}\right)$ was obtained in weed free plot which statistically at par with halauxifen + florasulam + polyglycol $\left(5151 \mathrm{~kg} \mathrm{ha}^{-1}\right)$, halauxifen + florasulam + carfentrazone + surfactant $\left(5117 \mathrm{~kg} \mathrm{ha}{ }^{-1}\right), 2,4-\mathrm{D} \mathrm{E}+$ carfentrazone, metsulfuron + carfentrazone + surfactant, 2, 4-D Na + carfentrazone and metsulfuron + surfactant. 
Table.1 Effect of herbicides on crop stand, plant height and plant dry matter accumulation at different crop growth stages

\begin{tabular}{|c|c|c|c|c|c|c|c|c|c|}
\hline \multirow[t]{2}{*}{ Treatments } & \multirow{2}{*}{$\begin{array}{c}\begin{array}{c}\text { Crop stand } \\
\text { (per metre row } \\
\text { length) }\end{array} \\
15 \text { DAS }\end{array}$} & \multicolumn{4}{|c|}{ Plant height (cm) } & \multicolumn{4}{|c|}{$\begin{array}{c}\text { Dry matter accumulation }\left(\mathrm{g} \mathrm{m}^{-1} \text { row }\right. \\
\text { length) }\end{array}$} \\
\hline & & 30 DAS & $\begin{array}{c}60 \\
\text { DAS }\end{array}$ & $\begin{array}{c}90 \\
\text { DAS }\end{array}$ & $\begin{array}{c}\text { At } \\
\text { harvest }\end{array}$ & $\begin{array}{c}30 \\
\text { DAS }\end{array}$ & 60 DAS & 90 DAS & $\begin{array}{c}\text { At } \\
\text { harvest }\end{array}$ \\
\hline $\begin{array}{l}\text { T}_{1} \text { - Halauxifen+ Florasulam+ } \\
\text { Polyglycol }\end{array}$ & 42.33 & 11.87 & 41.33 & 84.27 & 89.47 & 55.33 & 160.00 & 269.43 & 325.00 \\
\hline$T_{2^{-}}$Metsulfuron+ Surfactant & 41.67 & 11.60 & 39.33 & 82.27 & 86.53 & 53.67 & 146.00 & 250.00 & 313.00 \\
\hline $\mathbf{T}_{3}$ - Carfentrazone & 42.33 & 11.93 & 36.03 & 81.80 & 86.00 & 54.00 & 144.67 & 247.84 & 309.00 \\
\hline 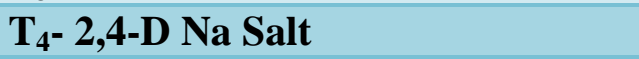 & 42.00 & 11.60 & 40.13 & 84.07 & 87.53 & 53.33 & 142.67 & 245.00 & 308.00 \\
\hline$T_{5}-2,4-D$ Ester & 42.33 & 10.73 & 39.07 & 81.33 & 86.07 & 53.33 & 145.33 & 248.00 & 310.00 \\
\hline $\begin{array}{l}\text { T6- Metsulfuron+ Carfentrazone + } \\
\text { Surfactant }\end{array}$ & 44.00 & 11.00 & 39.47 & 82.60 & 86.13 & 54.38 & 150.67 & 254.63 & 316.00 \\
\hline $\mathrm{T}_{7-}$ 2,4-D Na+ Carfentrazone & 42.33 & 10.80 & 38.95 & 82.00 & 88.87 & 52.67 & 149.33 & 251.49 & 315.00 \\
\hline$T_{8^{-}}$2,4-D E+ Carfentrazone & 42.00 & 11.13 & 39.60 & 82.73 & 88.20 & 54.00 & 155.33 & 261.67 & 320.00 \\
\hline $\begin{array}{l}\text { T9- Halauxifen+ Florasulam+ } \\
\text { Carfentrazone + Surfactant }\end{array}$ & 42.00 & 12.00 & 40.87 & 83.40 & 89.20 & 55.67 & 155.67 & 264.77 & 320.00 \\
\hline$T_{10}$ - Weedy check & 40.67 & 11.40 & 33.60 & 76.00 & 79.53 & 55.33 & 110.67 & 202.65 & 230.00 \\
\hline$T-11$ Weed free & 42.33 & 10.73 & 41.40 & 84.27 & 89.60 & 55.00 & 161.33 & 272.80 & 330.00 \\
\hline SEm \pm & 1.23 & 0.52 & 1.59 & 2.45 & 2.74 & 1.71 & 6.55 & 10.17 & 11.92 \\
\hline $\mathrm{CD}(\mathrm{P}=0.5)$ & 3.62 & 1.54 & 4.69 & 7.22 & 8.09 & 5.05 & 19.31 & 30.00 & 35.18 \\
\hline
\end{tabular}


Table.2 Effect of herbicides on total tillers 0.5 metre row length and growth kinetics at different growth stages

\begin{tabular}{|c|c|c|c|c|c|c|c|c|}
\hline \multirow[t]{2}{*}{ Treatments } & \multicolumn{4}{|c|}{ Total tillers 0.5 metre row length } & \multicolumn{2}{|c|}{ RGR ( g g $^{-1}$ day $\left.^{-1}\right)$} & \multicolumn{2}{|c|}{ CGR $\left(\right.$ g m$^{-2}$ day $\left.^{-1}\right)$} \\
\hline & $\begin{array}{c}\text { 30 } \\
\text { DAS }\end{array}$ & $\begin{array}{c}\text { 60 } \\
\text { DAS }\end{array}$ & $\begin{array}{c}90 \\
\text { DAS }\end{array}$ & $\begin{array}{c}\text { At } \\
\text { harvest }\end{array}$ & $\begin{array}{l}30-60 \\
\text { DAS }\end{array}$ & $\begin{array}{l}60-90 \\
\text { DAS }\end{array}$ & $\begin{array}{l}30-60 \\
\text { DAS }\end{array}$ & $\begin{array}{l}60-90 \\
\text { DAS }\end{array}$ \\
\hline $\begin{array}{l}\text { T1- Halauxifen+ Florasulam+ } \\
\text { Polyglycol }\end{array}$ & 26.00 & 47.00 & 60.00 & 66.00 & 0.035 & 0.017 & 174.44 & 182.39 \\
\hline $\mathbf{T}_{2}$ - Metsulfuron+ Surfactant & 25.00 & 42.00 & 55.00 & 60.00 & 0.033 & 0.018 & 153.89 & 173.33 \\
\hline$T_{3}$ - Carfentrazone & 24.00 & 40.30 & 53.00 & 58.00 & 0.033 & 0.018 & 151.11 & 171.96 \\
\hline $\mathrm{T}_{4^{-}}$2,4-D Na Salt & 24.00 & 41.03 & 52.00 & 57.00 & 0.033 & 0.018 & 148.89 & 170.56 \\
\hline$T_{5}=$ 2,4-D Ester & 25.00 & 42.00 & 54.00 & 59.00 & 0.033 & 0.018 & 153.33 & 171.11 \\
\hline $\begin{array}{l}\text { T6- Metsulfuron+ Carfentrazone + } \\
\text { Surfactant }\end{array}$ & 27.00 & 44.00 & 57.48 & 63.00 & 0.034 & 0.018 & 160.47 & 173.28 \\
\hline $\mathrm{T}_{7}-2,4-\mathrm{D} \mathrm{Na}+$ Carfentrazone & 26.00 & 42.00 & 56.00 & 62.00 & 0.035 & 0.017 & 161.11 & 170.27 \\
\hline$T_{8^{-}}$2,4-D E+ Carfentrazone & 27.00 & 45.00 & 57.55 & 64.00 & 0.035 & 0.017 & 168.89 & 177.22 \\
\hline $\begin{array}{l}\text { T9- Halauxifen+ Florasulam+ }_{\text {9 }} \text { + } \\
\text { Carfentrazone + Surfactant }\end{array}$ & 26.91 & 47.00 & 58.67 & 65.00 & 0.034 & 0.018 & 166.67 & 181.83 \\
\hline$T_{10^{-}}$Weedy check & 27.00 & 35.00 & 47.00 & 52.00 & 0.023 & 0.020 & 92.22 & 153.30 \\
\hline$T-11$ Weed free & 26.93 & 50.00 & 61.67 & 67.00 & 0.036 & 0.018 & 177.22 & 185.78 \\
\hline SEm \pm & 1.08 & 1.93 & 2.40 & 2.97 & 0.002 & 0.002 & 11.25 & 23.03 \\
\hline $\mathrm{CD}(\mathrm{P}=0.5)$ & 3.18 & 5.70 & 7.07 & 8.77 & 0.006 & 0.007 & 33.20 & 67.95 \\
\hline
\end{tabular}


Table.3 Visual phytotoxicity scoring on crop at different growth stages

\begin{tabular}{|c|c|c|c|c|}
\hline \multirow[t]{3}{*}{ Treatment } & \multicolumn{4}{|c|}{ Visual rating } \\
\hline & \multicolumn{2}{|c|}{ Leaf necrosis } & \multicolumn{2}{|c|}{ Yellowing of leaf } \\
\hline & $\begin{array}{c}5 \text { days after } \\
\text { spray }\end{array}$ & $\begin{array}{c}10 \text { days after } \\
\text { Spray }\end{array}$ & $\begin{array}{c}5 \text { days after } \\
\text { spray }\end{array}$ & $\begin{array}{c}10 \text { days after } \\
\text { Spray }\end{array}$ \\
\hline$T_{1}$ - Halauxifen+ Florasulam+ Polyglycol & 0 & 0 & 0 & 0 \\
\hline$T_{2}$ - Metsulfuron+ Surfactant & 0 & 0 & 0 & 0 \\
\hline $\mathbf{T}_{3^{-}}$Carfentrazone & 0 & 5 & 0 & 4 \\
\hline$T_{4-}$ 2,4-D Na Salt & 0 & 0 & 0 & 0 \\
\hline$T_{5-2}$ 2,4-D Ester & 0 & 0 & 0 & 0 \\
\hline 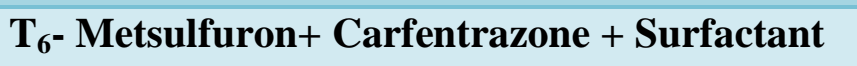 & 0 & 3 & 0 & 3 \\
\hline $\mathrm{T}_{7-}$ 2,4-D Na+ Carfentrazone & 0 & 3 & 0 & 3 \\
\hline $\mathrm{T}_{8^{-}}$2,4-D E+ Carfentrazone & 0 & 2 & 0 & 3 \\
\hline $\begin{array}{l}\text { T9- Halauxifen+ Florasulam+ Carfentrazone }+ \\
\text { Surfactant }\end{array}$ & 0 & 1 & 0 & 2 \\
\hline $\mathbf{T}_{10^{-}}$Weedy check & 0 & 0 & 0 & 0 \\
\hline$T_{-11}$ Weed free & 0 & 0 & 0 & 0 \\
\hline
\end{tabular}


Table.4 Effect of herbicides on yield and harvest index

\begin{tabular}{|c|c|c|c|c|}
\hline \multirow[t]{2}{*}{ Treatments } & \multicolumn{3}{|c|}{ Yield $\left(\mathrm{kg} \mathrm{ha}^{-1}\right)$} & \multirow{2}{*}{$\begin{array}{c}\text { Harvest } \\
\text { index } \\
(\%)\end{array}$} \\
\hline & Grain & Straw & Biological & \\
\hline$T_{1}$ - Halauxifen+ Florasulam+ Polyglycol & 5151 & 7264 & 12415 & 41.49 \\
\hline $\mathbf{T}_{2}$ - Metsulfuron+ Surfactant & 4930 & 6821 & 11751 & 41.96 \\
\hline $\mathbf{T}_{3}$ - Carfentrazone & 4587 & 6689 & 11277 & 40.69 \\
\hline$T_{4}-2,4-D$ Na Salt & 4606 & 6743 & 11350 & 40.61 \\
\hline$T_{5-1}$ 2,4-D Ester & 4630 & 6726 & 11356 & 40.76 \\
\hline$T_{6}$ - Metsulfuron+ Carfentrazone + Surfactant & 5021 & 7063 & 12085 & 41.55 \\
\hline $\mathrm{T}_{7}-$ 2,4-D Na+ Carfentrazone & 5015 & 6967 & 11982 & 41.85 \\
\hline T $_{8}$ - 2,4-D E+ Carfentrazone & 5117 & 7050 & 12167 & 42.06 \\
\hline $\begin{array}{l}\text { T9- Halauxifen+ Florasulam+ Carfentrazone + } \\
\text { Surfactant }\end{array}$ & 5117 & 7220 & 12337 & 41.31 \\
\hline$T_{10}$ - Weedy check & 4097 & 6341 & 10438 & 39.30 \\
\hline$T-11$ Weed free & 5287 & 7395 & 12682 & 41.68 \\
\hline SEm \pm & 174.70 & 207.99 & 252.44 & 1.18 \\
\hline $\mathrm{CD}(\mathrm{P}=0.5)$ & 515.38 & 613.56 & 744.70 & 3.47 \\
\hline
\end{tabular}

Table.5 Correlation coefficient $(r)$ and regression equation $(Y=a+b X)$ between various weed and crop parameters

\begin{tabular}{|l|l|l|l|}
\hline $\begin{array}{l}\text { Dependent } \\
\text { variable (Y) }\end{array}$ & Independent variable $(\mathbf{X})$ & $\begin{array}{l}\text { Correlation } \\
\text { coefficient } \\
(\mathbf{r})\end{array}$ & $\begin{array}{l}\text { Regression equation } \\
(\mathbf{Y}=\mathbf{a}+\mathbf{b X})\end{array}$ \\
\hline Grain yield & Plant dry matter at 60 DAS & $0.981^{* *}$ & $\mathrm{Y}=1350.80+23.86 \mathrm{X}$ \\
\hline Grain yield & Plant dry matter at 90 DAS & $0.977^{* *}$ & $\mathrm{Y}=476.67+17.45 \mathrm{X}$ \\
\hline Grain yield & Plant dry matter at harvest & $0.946^{* *}$ & $\mathrm{Y}=1394.99+11.25 \mathrm{X}$ \\
\hline $\begin{array}{l}\text { Plant dry matter } \\
\text { at 60 DAS }\end{array}$ & Plant height at 60 DAS & $0.888^{* *}$ & $\mathrm{Y}=-56.52+5.21 \mathrm{X}$ \\
\hline $\begin{array}{l}\text { Plant dry matter } \\
\text { at 90 DAS }\end{array}$ & Plant height at 90 DAS & $0.920^{* *}$ & $\mathrm{Y}=-363.48+7.47 \mathrm{X}$ \\
\hline $\begin{array}{l}\text { Plant dry matter } \\
\text { at harvest }\end{array}$ & Plant height at harvest & $0.938^{* *}$ & $\mathrm{Y}=-463.10+8.87 \mathrm{X}$ \\
\hline Straw Yield & Plant height at harvest & $0.863^{* *}$ & $\mathrm{Y}=-1121.80+92.58 \mathrm{X}$ \\
\hline Straw Yield & Plant DMA at 90 DAS & $0.924 * *$ & $\mathrm{Y}=3137.55+15.08$ \\
\hline Straw Yield & Plant DMA at harvest & $0.808^{* *}$ & $\mathrm{Y}=4099.79+9.18 \mathrm{X}$ \\
\hline
\end{tabular}


Fig.1 Effect of herbicides on plant dry matter accumulation $\left(\mathrm{g} \mathrm{m}^{-1}\right.$ row length) at different growth stages




The array of increase ranged from 5.5 (under carfentrazone) to 16.6 per cent (under weed free) over weedy check. The maximum straw produced by weed free plot (7395 kg ha-1) which statistically at par with halauxifen + florasulam + polyglycol, halauxifen + florasulam + carfentrazone + surfactant, 2, 4$\mathrm{D} \mathrm{E}+$ carfentrazone, metsulfuron+ carfentrazone + surfactant, 2, 4-D Na + carfentrazone and metsulfuron + surfactant.

It is well established fact that least crop weed competition during critical phases of crop growth exerts an important regulation function on complex process of yield formation due to better availability of water, space and nutrient. The results corroborate the findings of Mahmoud et al., (2016).

\section{Correlation study}

The correlation studies also showed a wellestablished significant negative correlation between crop yield and chenopodium album weed dry matter at 30,60 days after spray and at harvest with the respective values of $r=-$ 0.89, -0.92 and-0.98 (Table 5 and Fig. 1).

The maximum crop dry matter accumulation (330 $\mathrm{g} \mathrm{metre}^{-1}$ row length) was obtained in weed free plot followed by applying halauxifen + florasulam + polyglycol $(325 \mathrm{~g}$ metre $^{-1}$ row length). The maximum grain yield (5287 $\mathrm{kg} \mathrm{ha}^{-1}$ ) was acquired in weed free followed by controlling weeds by halauxifen + florasulam + polyglycol (5151 $\mathrm{kg} \mathrm{ha}^{-1}$ ).

The highest biological yield (12682 $\mathrm{kg} \mathrm{ha}^{-1}$ ) was exhibited in weed free followed by halauxifen + florasulam + polyglycol $(12415$ $\mathrm{kg} \mathrm{ha}^{-1}$ ), while maximum straw yield (7395 $\mathrm{kg} \mathrm{ha}{ }^{-1}$ ) recorded in weed free followed by application halauxifen + florasulam + polyglycol (7264 $\left.\mathrm{kg} \mathrm{ha}^{-1}\right)$.

\section{References}

Braun, H.J., Atlin, G. and Payne, T. (2010). Multi location testing as a tool to identify plant response to global climatic change. In: Climate Change and Crop Production CABI, London, UnnitedKingdom.pp- 8-9.

Carlos Campillo, Rafael Fortes and Maria delHenarPrieto. (2012). Solar radiation effect on crop production. In: Solar Radiation, Elisha B. Babatunde (Ed.), ISBN: 978-953-51-0384-4, Intech, (http://www.intechopen.com/bppks/sola r-radiation/solar-radiation-effect-oncrop-production).

Commissionerate of Agriculture, Crop-wise Area, Production and Yield of various principal crops Second Advance Estimates of Kharif 2019 \& First Advance Estimates of Rabi 2019-20, Rajasthan-Jaipur

DACFW. (2018-19). Annual Report, Department of Agriculture, Cooperation \& Farmers Welfare, Ministry of Agriculture, Cooperation \& Farmers Welfare, Government of India, New Delhi.

Dixon, J., Braun, H.J. and Crouch, J.H. (2009). Overview: Transitioning wheat research to serve future needs of developing world. In: Wheat Facts and Futures CYMMIYT, Mexico pp- 1-25.

Duncon, W. G. (1971). Leaf angle, leaf area and canopy photosynthesis. Crop Science 11(4): 482-485.

Fakkar, A.A.O. and Amin, I.A. (2012). Integration between sowing methods and mechanical weed control and their effect on wheat productivity. Australian Journal of Basic and Applied Science 6(13):5 19-529.

Gupta, O. P. (2007). Modern weed management. Third revised edition. Agribios (India) Publication.pp-130, Appendix 11. 
Harper, J. I. (1977). The population biology of plants. Academic Press, London, UK.

Mahmoud, S. M., Soliman, F. S. and Elsheik, M. (2016). Combination of halauxifen methyl + florasulam with other grassy herbicides against complex weed flora in wheat (Triticum aestivum).Journal of Plant Protection and Pathology 7(5): 315-320.

Marwat, K. B., Mahammad, S., Zahid, H., Gul, B. and Rashid, H. (2008). Study of various weed management practices for weed control in wheat under irrigated conditions. Pakistan Journal of Weed Science Research 14 (1-2): 1-8.

Singh, R., Singh, P., Singh, V. K., Singh, V. P. and Pratap, T. (2012). Effect of different herbicides on weed dry matter and yield of wheat. International Agronomy Congress 2: 138-139.

Swaminathan, M.S. and Bhavani, R.V. (2013). Food production and availability - Essential prerequisites for sustainable food security. Indian Journal of Medicinal Research, 138: 383-391.

Swanton, C.J., Nkoa, R. and Blackshaw, R.E. (2015). Experimental methods for cropweed competition studies. Weed Science 63(1): 2-11.

Yaduraju, N.T. and Das, T.K. (2002). Bioefficacy of metsulfuron- methyl and 2, 4-D on Canada thistle. Indian Journal of Weed Science 34(1\&2):110-111.

\section{How to cite this article:}

Shivran. A. C., Sarita, J. Choudhary and Jitendra Singh Bamboriya. 2020. Effect of Different Herbicides on Growth and Yield of Wheat (Triticum aestivum L.). Int.J.Curr.Microbiol.App.Sci. 9(04): 438-448. doi: https://doi.org/10.20546/ijcmas.2020.904.053 